hemoglobin concentrations and an increased risk of cardiovascular events.

To examine the influence of different hemoglobin targets on the outcomes of patients with chronic kidney disease receiving recombinant human erythropoietin, Phrommintikul et al. conducted a meta-analysis involving 5,143 patients from nine randomized trials. Only trials that recruited at least 100 patients and had a minimum follow-up of 12 weeks were included in the analysis. The risk of all-cause mortality was significantly greater in the patients assigned to a higher hemoglobin target (120$160 \mathrm{~g} / \mathrm{l}[12-16 \mathrm{~g} / \mathrm{dl}])$ than in those assigned to a lower hemoglobin target (90-120 g/l [9-12 g/dl]; relative risk 1.17, 95\% $\mathrm{Cl} 1.01-1.35 ; P=0.031$ ), as was the risk of arteriovenous access thrombosis in the six studies that included patients on hemodialysis (relative risk 1.34, 95\% Cl 1.16$1.54 ; P=0.0001)$. The risk of poor blood pressure control was significantly increased in the higher hemoglobin target group when a fixed effects model was used for analysis (4 studies; $P=0.004)$, but not when a random effects model was used $(P=0.075)$. There was no significant difference in the risk of myocardial infarction between the high and low hemoglobin target groups ( 7 studies; $P=0.88$ ).

On the basis of these results, the authors suggest that future clinical practice guidelines should set an upper limit for target hemoglobin concentration during erythropoietin therapy.

Original article Phrommintikul A et al. (2007) Mortality and target haemoglobin concentrations in anaemic patients with chronic kidney disease treated with erythropoietin: a meta-analysis. Lancet 369: 381-388

\section{Ionic dialysance shows promise for quantification of dialysis dose in acute renal failure}

For patients with acute renal failure (ARF) receiving intermittent hemodialysis (IHD), quantification of the 'dose' of dialysis delivered is problematic. Fractional dialysate sampling is the gold standard; however, this type of sampling is labor-intensive and, therefore, not routinely used. Dialysis dose is more frequently estimated on the basis of urea kinetics as $K t / V$. This formula has only been validated in patients with chronic renal failure, and assumes a steady state and constant distribution of urea. The relevance of the $K t / V$ formula in ARF is, therefore, doubtful, as these assumed conditions are perturbed by hemodynamic instability and multiple interruptions to therapy. Ionic dialysance-calculated automatically by the dialysis unit in real time from the dialysate conductivity-is an alternative method of quantifying urea clearance. It is predicated on the similar transfer characteristics of ions and urea during IHD. Ridel et al. have demonstrated the applicability of this method to ARF in a population of 31 patients treated with IHD.

During each patient's initial IHD session, the researchers compared the dose of dialysis delivered as calculated by ionic dialysance, with the dose of dialysis delivered according to fractional dialysate sampling. A highly significant linear correlation was found between the two methods of dose determination $(r=0.96 ; P<0.01)$, and Bland-Altman analysis demonstrated very good limits of agreement $(-2.21,6.41)$. Dialysis efficiency had no effect on this association.

Original article Ridel C et al. (2007) Ionic dialysance: a new valid parameter for quantification of dialysis efficiency in acute renal failure? Intensive Care Med 33: 460-465

\section{Falls in systolic blood pressure during hemodialysis relate to good short-term outcome}

A drop in systolic blood pressure (SBP) of at least $10 \mathrm{mmHg}$ during maintenance hemodialysis is associated with fewer hospitalizations and deaths, report Inrig et al. These authors performed a secondary analysis of 443 patients with end-stage renal disease who were enrolled in the randomized Crit-Line Intradialytic Monitoring Benefit Study.

SBP fell by at least $10 \mathrm{mmHg}$ during hemodialysis in 230 patients. These individuals were younger, on fewer blood pressure medications, and had higher predialysis blood pressure, serum creatinine levels and dry weights than those whose SBP did not fall. After controlling for these and other factors, the likelihood of non-access-related hospitalization or death at 6 months was significantly lower in the group with a hemodialysis-related fall in SBP than in those whose SBP changed by less than $10 \mathrm{mmHg}(n=150$; odds ratio $1.85,95 \% \mathrm{Cl}$ $1.15-2.98)$ or rose by at least $10 \mathrm{mmHg}(n=58$; odds ratio $2.17,95 \% \mathrm{Cl} 1.13-4.15)$. In adjusted models, every $1 \mathrm{mmHg}$ hemodialysis-related 